\title{
Modelling of tribo-electrostatic separation for industrial by-products recycling
}

\author{
F. Cangialosi, F. Crapulli, G. Intini, L. Liberti \& M. Notarnicola \\ Department of Environmental Engineering and Sustainable Development, \\ Technical University of Bari, Taranto, Italy
}

\begin{abstract}
Tribo-electrostatic separation is a dry technology which allows the elimination of impurities from industrial wastes on the basis of their surface charging characteristics. In this paper results of investigations aiming to numerically simulate the tribo-electrostatic separation of fine particles for industrial by-products recycling are described. After charging in a pneumatic transport line, the mixture of the particles to be separated is injected in a separation chamber where a DC electric field is created using two parallel plate electrodes. The particle/gas flow inside the chamber was simulated using an extended commercial computational fluid dynamics (CFD) code. The three-dimensional turbulent flow was calculated. Based on the Lagrangian approach, the trajectories of the powder particles ( $<100$ microns) were modelled considering electric and aerodynamic forces. Comparison of the simulation results with experiments carried out with a bench-scale separation unit are presented for the case of silica beads, whose electric characteristics resemble those of coal fly ashes. The effects of particle charge, electric field and injection velocity were investigated, revealing that a proper choice of separator geometry and flow parameters allows unwanted fine particles in industrial wastes like fly ash to be efficiently removed. Keywords: powder wastes, tribo-electrostatic separation, computational fluid dynamics (CFD).
\end{abstract}

\section{Introduction}

For the sustainable development of our society, recycling of municipal and industrial wastes must continue to be pursued. Many industrial wastes (combustion fly ashes, residues of surface finishing process in soft metallurgy, commingled plastic waste from electric/electronic equipments, etc.) are in 
powder or granulated form, having a grain size below $1 \mathrm{~mm}$. Nearly all the valuable components in the industrial waste can be reused by subjecting the material to advanced treatment using effective methods to separate and purify each component. In the case of coal fly ash, more than $90 \%$ of the material can be re-used for cement and concrete production as long as the carbon content in the ash is below $5 \%$, so that separation technologies are applied to meet the requirements in terms of product purity, whereas $\mathrm{ABS}$, PS and PP were recovered from a plastic mixtures with recoveries above $73.0 \%$ and a grade of $92.1 \%, 84.9 \%$ and $90.0 \%$, respectively $[1,2]$. Interest in electrostatic separation is increasing because it appears to be the first dry method, which does not require wastewater treatment processes, to separate particles with alike density and electrical conductivity. Tribo-electrostatic separation is a technology developed in the field of the mining engineering which allows to separate materials on the basis of their surface charging characteristics: for dissimilar solids, which are initially uncharged, a transfer of a small electrical charge takes place from one material to the other as they make contact, where one becomes negative and the other positive. In the pneumatic tribo-electrostatic separation, particles to be separated are air-transported in pipelines where they collide with the inner wall of the tube and become positively and negatively charged; particles are then injected in a separation reactor where an electric field is applied to separate the particles. Although this technology works well in the separation of relatively coarse materials (diameter above $1 \mathrm{~mm}$ ), much work has still to be done for making tribo-electrostatic separation effective in fine particles separation.

The main goal of the research activities here reported was to experimentally investigate the process parameters which mostly affect the separation process of powders and gain fundamental information for proper system design and operation by means of Computational Fluid Dynamics (CFD).

\section{Experimental equipment}

In order to elucidate the effect each parameter has on the system performance and avoid the arise of secondary effects related to particle-particle interactions, no mixtures were used in the separation tests: a single material (glass beads, average diameter 65 microns) was tested and the trajectories of the particles in the separation chambers at different voltages (negative and positive) and injection velocities were studied with CFD. A schematic of the bench-scale apparatus, having maximum throughput of $20 \mathrm{~kg} / \mathrm{h}$, employed for triboelectrostatic separation tests is shown in Figure 1. A small amount of glass beads was placed in a vibratory feeder contained in a sealed tank. Falling from the feeder, the particles are transported through a $6.35 \mathrm{~mm}$ diameter stainless steel charging tube by air at a flow rate between 7 and $20 \mathrm{~m} / \mathrm{s}$ controlled by a rotameter. The charger tube forms three loops that increase the number of collisions between particles and wall pipe, thus establishing a charge polarity on glass beads.

Increasing the length and turbulence within this charger section obviously enhances the chance of charging for particle-particle collisions. Injected with a 
diffuser through the flow-straighteners, the air-particle mixture enters the separation chamber ( $35 \mathrm{~cm}$ long) containing the electrodes made by two copper plates attached to its opposite side walls. Under the electric field generated by a high voltage power supply, the charged particles are deflected towards the electrodes according to their polarity. A flow splitter then convoys the two streams of particle-laden flows through two cyclones from which two product streams were collected and the mass of glass beads was measured. The injection velocity in all the experiments was set to a constant value and the velocities at the cyclones were adjusted in order to set a balanced flow between the product outlets, unless explicitly stated; the electric field ranged from $-2 \mathrm{kV} / \mathrm{cm}$ to $2 \mathrm{kV} / \mathrm{cm}$. The ratio between the injection velocity and the velocity measured at the cyclones was varied between 0.1 and 3 , in order to study the effect of co-flow air entering from the flow-straighteners, whose primary role is to reduce the turbulence in the separation chamber.

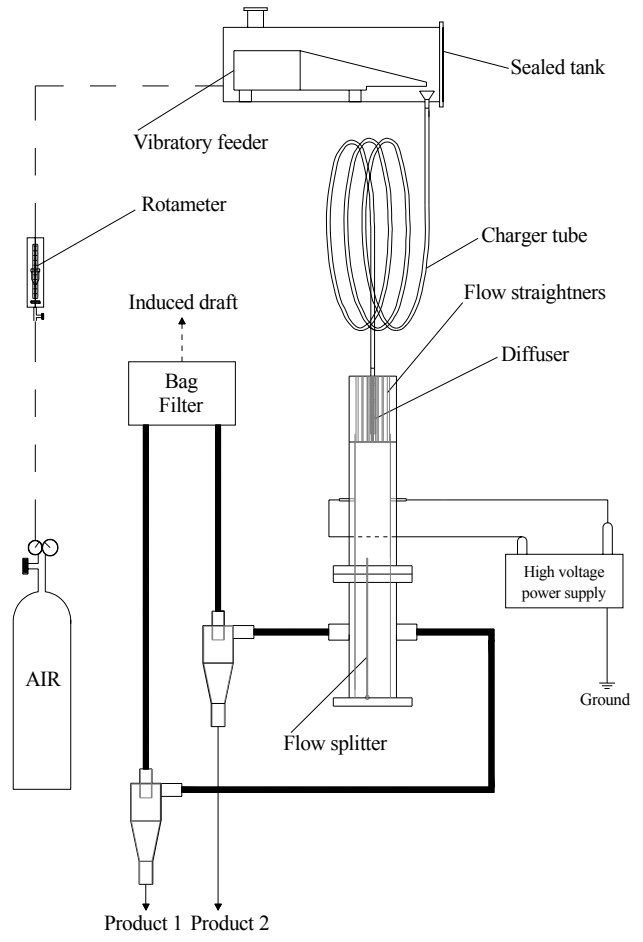

Figure 1: Schematic of the experimental setup.

\section{Numerical method}

\subsection{Gas flow}

The experimental results (mass of glass beads recovered) were compared to those obtained with the use of CFX, a commercial CFD code. The separation 
chamber was discretized in one million of elements with an unstructured mesh and the Navier-Stokes equations with a standard k- $\varepsilon$ model of turbulence were solved for the air flow distribution. The three-dimensional turbulent flow field was calculated in a computational domain with the size of $37.5 \times 50 \times 350 \mathrm{~mm}^{3}$ consisting of the separation chamber. A hybrid unstructured mesh of different elements (from 500,000 up to 1,500,000) is used in this study. Mass and momentum balance equations are integrated over the elements of the unstructured mesh [3]. The standard model k- $\varepsilon$ was employed to simulate the turbulent motion with a sufficient accuracy and low CPU time cost. The following assumptions for the model were taken: (1) the fluid is incompressible, (2) the turbulent flow field is isotropic, (3) the boundary conditions of smooth wall and no slip were chosen. The two-phase flow enters the separator at $7 \mathrm{~m} / \mathrm{s}$ through a slit $3 \mathrm{~mm}$ wide thus creating a plane jet. A honeycomb structure at the inlet of the separation chamber allows the air at atmospheric pressure to be sucked into the cell were a slight negative pressure is set by means of a vacuum pump. The simulations of the flow field were carried out investigating a range of velocity at the cyclones outlet ranging between 1.4 and $35 \mathrm{~m} / \mathrm{s}$.

\subsection{Particle motion and electric field}

Particle tracking inside the separation chamber allows trajectories of the charged particles to be followed from the diffuser to the cyclones outlet. The assumption of dilute flow was made during the simulation, so that influence of particle motion on gas flow and particle-particle collisions were neglected: the particle tracking routine employed was than based on the hypothesis of one-way coupling between gas and particles $[4,5]$. Particles are injected in the separator with the same velocities as local air flow and with several angles $\varphi$ from the direction of the $z$-axis. We assumed that $\varphi$ is expressed by a normal distribution with average $\varphi_{\mathrm{av}}=0$ and standard deviation $\sigma_{\varphi}$. The following steps could be undertaken to carry out the simulation of particle motion in the electric field generated by the parallel plate electrodes inside the separator:

1. solve the electric field neglecting the space charge $\rho_{E}$ created by the charged particles using Laplace equation with the proper boundary conditions;

2. calculate the charged particle trajectories with the Lagrangian approach considering aerodynamic and electrical forces acting on the particles:

$$
m_{p} \frac{d v_{p}}{d t}=\frac{1}{8} \pi \rho d^{2} C_{D}\left|v_{f}-v_{p}\right|\left(v_{f}-v_{p}\right)+\frac{\pi d^{3} \rho_{f}}{6} \frac{d v_{f}}{d t}+\frac{1}{6} \pi d^{3}\left(\rho_{p}-\rho_{f}\right) g+F_{E}
$$

where $v_{p}$ and $v_{f}$ are the particle and fluid velocity, respectively, $C_{D}$ is the drag coefficient [5] and $F_{E}$ is the electric force $q E, q$ being the particle charge and $E$ the electric field;

3. calculate the space charge distribution $\rho_{E}$ inside the separator;

4. solve the electric filed by using the Poisson equation with the space charge obtained from the previous step;

5. repeat steps 2 to 4 until solution is convergent. 
This numerical technique was originally proposed by Elmoursi [6]. In our case only steps 1 to 3 were considered as the space charge was considered sufficiently small to not affect the electric field distribution.

\section{Results and discussion}

\subsection{Gas flow field}

In order to evaluate the accuracy of the turbulence model in predicting the gas flow field, a grid-independence analysis was carried out with a number of grid elements ranging from 850,000 up to $1,500,000$. Two control lines within the separator were chosen to compare the simulated mean gas velocity component along the $z$-axis $\left(v_{z}\right)$, varying the number of elements, with experimental data obtained by other authors. The first line starts from the diffuser along the central axis of the separator (z-axis) to a distance $0.25 \mathrm{~m}$ downwards; the other line was set crossflow ( $x$-axis) at $0.10 \mathrm{~m}$ downwards from the diffuser. In order to compare the results, the excess velocity $\left(\mathrm{v}_{\mathrm{z}}-\mathrm{v}_{\text {coflow }}\right)$ was made dimensionless by dividing it to the excess velocity along the centreline $\left(\mathrm{v}_{\mathrm{z}, \mathrm{centreline}}-\mathrm{V}_{\text {coflow }}\right)$; the space scales were made dimensionless by dividing $z$ and $x$ to the diffuser width $D$ and the distance from the center-line at which the velocity decreases to $50 \%\left(r_{1 / 2}\right)$, respectively.

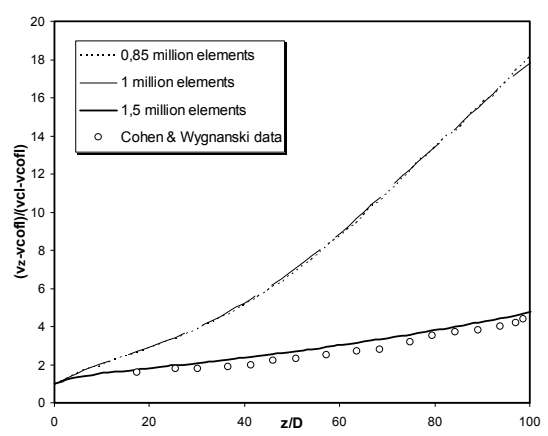

(a)

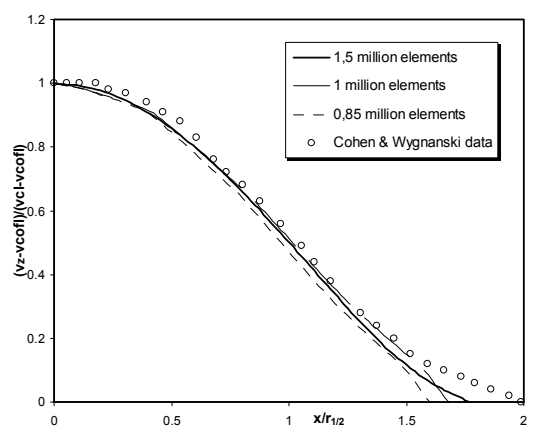

(b)

Figure 2: $\quad$ Comparison between mean gas velocity $\mathrm{v}_{\mathrm{z}}$ along the control lines in $z$-direction (a) and $x$-direction (b) with different number of elements and experimental data [7].

Figure 2 shows the results obtained from the grid-independence study: the data measured by Cohen and Wygnanski [7] are in good agreement with simulations of the gas flow field along the second control line (Figure 2(b)) even with the smallest numbers of elements considered in the simulations. Nevertheless, it was shown that 1,500,000 elements are required to model the process with sufficient accuracy along the first control line (Figure 2(a)). In all the simulations with particles, 1.5 million of elements were then used, which is 
the minimum number that allows one to obtain a good simulation with the standard k- $\varepsilon$ model of turbulence.

\subsection{Particles separation}

The model validation was carried out by comparing the experimental results obtained with the bench-scale tribo-electrostatic separator previously described, with the outcome of simulations were an appropriate choice of calibration parameter was made: it was surmised that the standard deviation $\sigma_{\varphi}$ of the particle injection angle $\varphi$ distribution was the most important calibration parameter. During the first validation trials the flow inside the chamber needed to be balanced by regulating the suction velocities at the cyclones, as the flow splitter was not set in the middle of the separator but $1.2 \mathrm{~cm}$ close to the left plate. Glass beads in the size range 40-70 $\mu \mathrm{m}$ were employed in the experiments. The charge distribution of glass beads was experimentally and theoretically obtained in other works $[8,9]$. The mass fraction of glass beads collected in the left cyclone (Figure 1) was compared to the number of particles leaving the separator form the same outlet divided to the total number of particles used in the simulation. The best agreement between measured $(22 \%)$ and calculated $(25 \%)$ mass fraction was obtained with the standard deviation $\sigma_{\varphi}$ of particle injection angle set to $2.2^{\circ}$. The distribution of different injection angles was reproduced by considering five particle classes having injection velocity in the $x$-axis between 0.6 and $0.6 \mathrm{~m} / \mathrm{s}$. When the electric field was set to $2 \mathrm{kV} / \mathrm{cm}$ (a positive value indicates that the left plate is positively charged), the majority of particles were deflected towards the positive electrode, as indicated by other studies confirming that most of the glass particles become negatively charged during pneumatic transport [8]. The following table summarizes the results obtained in the calibration procedure.

Table 1: Comparison between experimental and simulation results of particle mass fraction deflected towards the left electrode.

$$
\text { Mass fraction (\%) }
$$

Electric field $(\mathrm{kV} / \mathrm{cm})$ Experimental Theoretical

\begin{tabular}{ccccc}
\hline & Left plate & Right plate & Left plate & Right plate \\
0 & 78 & 22 & 75 & 25 \\
2 & 2 & 98 & 2 & 98 \\
\hline
\end{tabular}

After the first calibration step, a voltage-dependence study was carried out to evaluate the influence of electric field strength on particle collection efficiency.

In order to assess whether the calibration parameter was robust enough to adequately describe the particles motion, the suction velocities were adjusted to establish an unbalanced flow in the separator: since the standard deviation $\sigma_{\varphi}$ does not depend on the flow field, the value chosen in the previous experiments 
$\left(2.2^{\circ}\right)$ should have given the same agreement between measured and simulated results. The electric field was varied between $-2 \mathrm{kV} / \mathrm{cm}$ and $2 \mathrm{kV} / \mathrm{cm}$, where a negative value corresponds to a negative charge polarity on the left plate and the yield is calculate as the mass collected at the right outlet over the entire mass fed to the system (Figure 3(b)). In Figure 4 the outcomes of this analysis are shown.

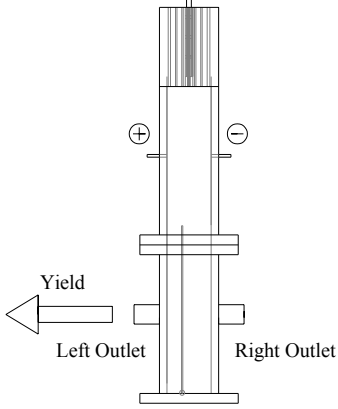

(a)

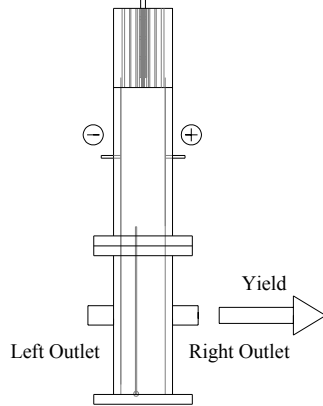

(b)

Figure 3: $\quad$ Schematic of the yield calculation for positive (a) and negative (b) electric field.

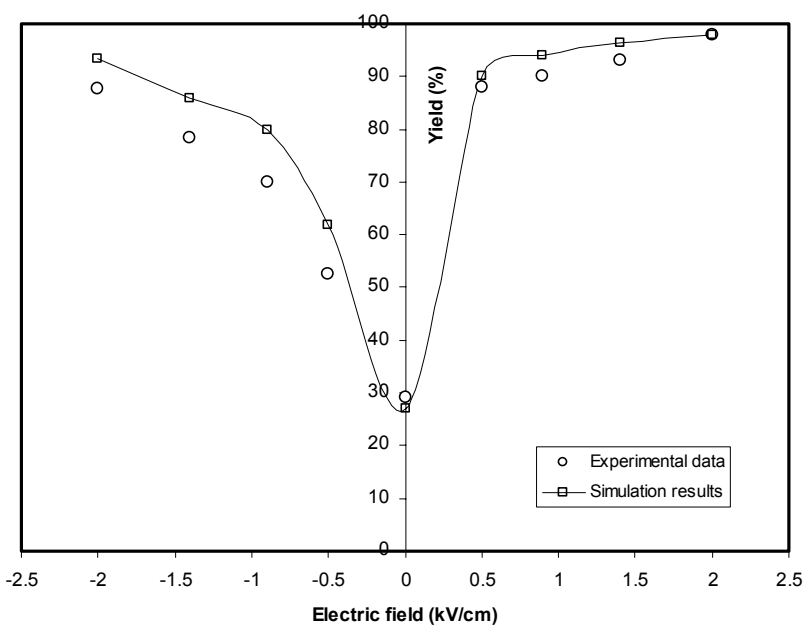

Figure 4: Comparison of yields between experimental tests and numerical simulations at different electric fields.

It must be pointed out that the good agreement between calculations and measured results without electric field remarks the adequacy of the calibration procedure of the standard deviation $\sigma_{\varphi}$, even with an unbalanced flow. When the electric field is increased until $2 \mathrm{kV} / \mathrm{cm}$, the yield increases to $98 \%$ while in the 
case of negative fields, a mass fraction of $87 \%$ was collected in the right cyclone $(\mathrm{E}=-2 \mathrm{kV} / \mathrm{cm})$. The asymmetry is the consequence of the fact that the flow splitter is set close to the left plate. Figure 5 shows the volume fraction distribution in the cross-flow direction ( $x$-axis) of the five particle size classes without electric field (Figure 5(a)) and with a negative field of $-0.9 \mathrm{kV} / \mathrm{cm}$ (Figure 5(b)).

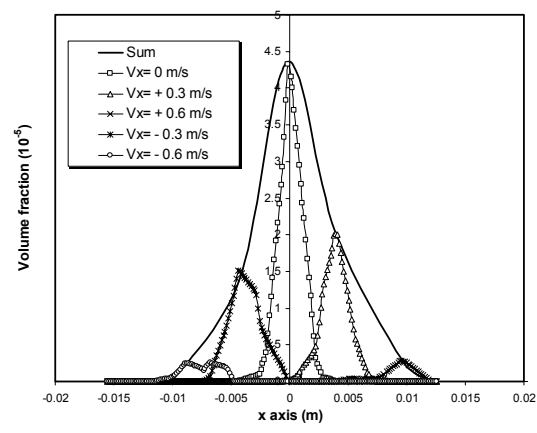

(a)

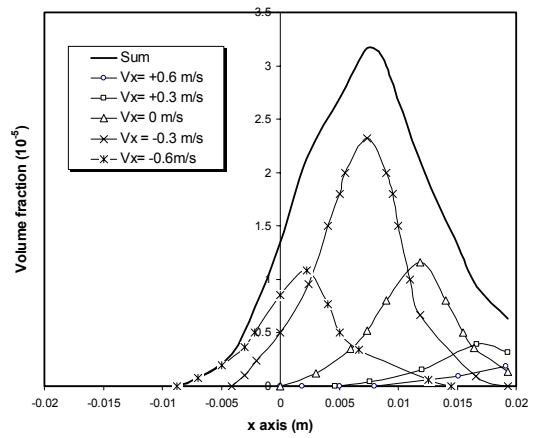

(b)

Figure 5: Volume fraction distribution of particles in the separator without electric field (a) and with an electric field of $-0.9 \mathrm{kV} / \mathrm{cm}(\mathrm{b})$.

Without electric field the particle distribution across the transversal section of the separator s not symmetric, due to the unbalanced flow field. With a negative electric field, the volume fraction distribution is significantly shifted towards the positive electrode (the right cyclone); some particles are however deflected towards the negative electrode because of either the initial horizontal velocity or the charge distribution which accounts for the fact that some particles can become positively charged in the transport line [9]. Besides the aspects related to particle movement due to electric field, another part of the investigation was focused on determining the effect of co-flow air in stabilizing the flow patterns in the separator. The lower the particle inertia, the more the effects of turbulence of the jet will be and, as a consequence, the particles are less likely to respond to electric force as compared to turbulent fluctuations. This occurrence could make tribo-electrostatic separation ineffective for small particles, unless turbulent eddies in the separator were dumped. The use of flow-straightners and the creation of a slight negative pressure in the separation chamber were experimentally found to have a great importance in the separation of low inertia particles by dumping the turbulent eddies [1, 8]. It was found that co-flow velocities slightly less than flow injection velocity cause only very small particles $(<5 \mu \mathrm{m})$ to be entrapped in the eddies, while heavier particles follow the mean flow structures, as it happens when the ratio co-flow to flow velocity is greater than one. To simulate the condition without co-flow (Figure 6(a)), no slip end smooth wall conditions were assumed for the separator lateral boundaries, while atmospheric pressure was set at the two outlets [10]. 
Figure 6(b) shows the streamlines inside the separation chamber with co-flow $10 \%$ greater than flow, which points out the remarkable reduction in eddies due to entrainment [11].

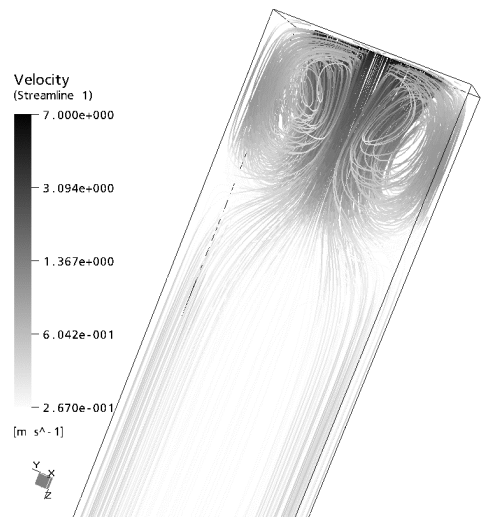

(a)

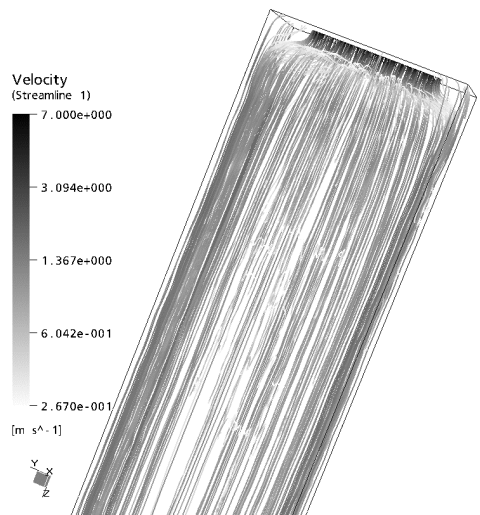

(b)

Figure 6: Streamlines of particle jet in the separation cell (side view) without co-flow (a) and with ratio coflow-to-flow set to 1.1 (b).

The presence of additional co-flow air reduces the dissipation of the jet due to viscous forces, so that the average fluid and particle axial velocity diminishes more slowly allowing then the fine particles to be attracted by the plates, contributing to an overall enhancement of separation performances.

\section{Conclusions}

Some fundamental issues related to the motion of tribo-charged particles in an electrostatic separator were addressed. Separation performances of fine powders (glass beads) were analyzed experimentally and theoretically by the Lagrangian simulation method. Simulation results agreed very well with all the experiments when the calibration parameter, the standard deviation $\sigma_{\varphi}$ of particle injection angle, was set to $2.2^{\circ}$. Once the model was calibrated, several simulations were carried out, with the aim of gain much fundamental understanding of particle motion in the separation chamber, which could not have been possible without computational fluid dynamics. First it was studied the evolution of volume fraction distribution of the particles in the separator due to the electric field and un-balanced flow. Then it was analyzed the importance of co-flow air related to mixing degree in the chamber (flow air to co-flow air ratio) and particles entrainment in turbulent eddies. The results show that in a free-fall separator, where no co-flow air is employed, particles re-circulate nearby the injection point, thus reducing the effect of electric force on the particle movement; on the other-hand, when the co-flow air is high enough to enhance the air entrainment 
in the separation chamber, the degree of mixing raises, the particle jet is more stable and particles are more easily deflected on the basis of their charge. Hence, this study confirms that tribo-electrostatic separation of fine particles deriving from industrial wastes is effective as long as the proper flow parameters and separator geometry are chosen: this occurrence opens new possibilities in the field of re-use of wastes made of fine particles by means of dry separation which has been halted to date by technological problems associated with free-fall separators.

\section{References}

[1] F. Cangialosi. Dry triboelectrostatic beneficiation of coal combustion fly ash. Ph.D. dissertation, Department of Environmental Engineering and Sustainable Development, Technical University of Bari, 2005.

[2] G. Dodbiba, A. Shibayama, T. Miyazaki, T. Fujita. Triboelectrostatic separation of ABS, PS and PP plastic mixture. Materials Transactions, 44, 161-166, 2003.

[3] S. Yuu, K. Ikeda, T. Umekage. Flow-field prediction and experimental verification of low Reynolds number gas-particle turbolent jets. Colloids and surfaces A, 109, 13-27, 1996.

[4] L.J.S. Bradbury \& J. Riley. The spread of a turbolent plane jet issuing into a parallel moving air stream. Journal of Fluid Mechanics, 27, 381-394, 1967.

[5] G. Gousbet, A. Berlemont. Eulerian and Lagrangian approaches for predicting the behaviour of discrete particles in turbulent flows. Progress in Energy and Combustion Science, 25, 133-159, 1998.

[6] A.A. Elmoursi \& G.S.P. Castle. Modelling of corona characteristics in a wire-duct precipitator using the charge simulation technique. IEEE Trans. Ind. Appl., 23, 95-102, 1987.

[7] J. Cohen \& I. Wygnanski. The evolution of instabilities in the axisymmetric jet. Part 1. The linear growth of disturbances near the nozzle. Journal of Fluid Mechanics, 176, 191-219, 1987.

[8] T. Li. An experimental study of particle charge and charge exchange related to triboelectrostatic beneficiation, Ph.D. dissertation, Department of Mechanical Engineering, University of Kentucky, Lexington, KY, 1999.

[9] F. Cangialosi, L. Liberti, M. Notarnicola, J.M. Stencel. Monte-Carlo simulation of pneumatic tribo-charging in two-phase flow for high-inertia particles, accepted for publication on Powder Technology.

[10] R.W. Schefer, V. Hartman, R.W. Dibble. Conditional sampling of velocity in a turbolent non-premixed propane jet. Sandia National Laboratories Report SAND87-8610, (1987).

[11] T.B. Nickels \& A.E. Perry. The turbolent co-flowing jet. Journal of Fluid Mechanics, 309, 157-182, 1996. 